\title{
CLASSICAL LOGICS FOR ATTRIBUTE-VALUE LANGUAGES
}

\author{
Jürgen Wedekind \\ Xerox Palo Alto Research Center \\ and \\ C.S.L.I. - Stanford University \\ USA
}

\begin{abstract}
This paper describes a classical logic for attributc-value (or feature description) languages which are used in unification grammar to describe a certain kind of linguistic object commonly called attribute-value structure (or feature structure). The algorithm which is used for deciding satisfiability of a foalure description is based on a restricted deductive closure construction for sets of literals (atomic formulas and negated atomic formulas). In contrast to the Kasper/Rounds approach (cf. [Kasper/Rounds 90]), we can handle cyclicity, without the need for the introduction of complexity norms, as in [Johnson 88] and [Beierle/Pletat 88]. The deductive closure construction is the direct proof-theoretic correlate of the congruence closure algorithm (cf. [Nelson/Oppen 80]), if it were used in attributevalue languages for testing satisfiability of finite sets of literals.
\end{abstract}

\section{Introduction}

This paper describes a classical logic for attribute-value (or feature description) languages which are used in unification grammar to describe a certain kind of linguistic object commonly called attribute-value structure (or fcature structure). From a logical point of view an attribute-value structure like e.g. the following (in matrix notation)

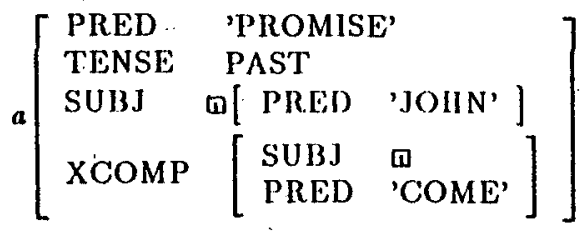

can be regarded as a graphical representation of a minimal model of a satisfiable feature description. If we assume that the attributes (in the example: PRED, TENSE, SUBJ, $\mathrm{XCOMP)}$ are unary partial function symbols and the values ( $a$, 'PROMISE', PAST, 'JOIIN', 'COME') are constants then the given feature structure represents graphically e.g. the minimal model of the following description:

PRED SUBJ $a \approx$ 'JOHN' \& TENSE $a \approx$ PAST \& PREDa $\approx$ 'PROMISE' \& SUBJa $\approx$ SUBJ XCOMPa \& PRED XCOMPa $\approx$ 'COME'.'

\footnotetext{
'Note that the terms are formed without using brackets. (Since all function symbols are unary, the introduction of brackets would
}

So, in the following attribite-value languages are regarded as quantifier-free sublanguages of classical first order languagez with equality whose (nonlogical) symbols are given by a sel ó unary partial function symbols (attributes) and a set of constants (atomic and complex values). The logical vocabulary includes all propositional connectives; negation is interpreted classically. ${ }^{2}$

For quantifier-free attribute-value languages $L$ we give an axiomatic or Hilberi type system $H_{A V}^{0}$ which simply results from an ordinary first order system (with partial function symbols), if its language were restricted to the vocabulary of $L$. According to requirements of the applications, axioms for the constantconsistency, constant/complex-consistency and acyclicity can be added to force these properties for the feature structures (models).

For deciding consistency (or satisfiability) of a fealure description, we assume first, that the conjunction of the formulas in. the feature description is converted to disjunctive normal form. Since a formula in disjunctive normal form is consistent, iff at least one of its disjuncts is consistent, we only need an algorithm for deciding consistency of finite sets of literals (atomic formulas or negated atomic formulas) $S$. In contrast to the reduction algorithms which normalize a set $S$ according to a complexity norm in a sequence of norm decreasing rewritc steps ${ }^{3}$ we use a restricted deductive closure algorithm for deciding the consistency of sets of literals." The restriction results from the fact that it is sufficient for deciding the consistency of $S$ to consider proofs of equations from $S$ with a certain subtern property. For the closure construction only those equations are derived from $S$ whose terms are sublerms of the terms occurring in the formulas of $S$. This guarantees that the construction terminates with a finite set of literals. The adequacy of this subterm property restriction, which was already shown for the number theoretic calculus $K$ in [Kreisel/Tait 61] by [Statman 74], is a necessary condition for the development of more efficient Cut-free Gentzen type systems for attribute-

not improve the readability essentially.) Therefore we write e.g. PRED SUBJa instead of PRED(SUBJ(a)).

${ }^{2}$ For intuitionistic negation cf. e.g. [Dawar/Vijay-Shanker 90] and [Langholm 89].

${ }^{3}$ Cf. e.g. [Kreisel/Tait 61], [Knuth/Bendix 70], and applied to attribute-value langunges [Johnson 88], [Beierlo/Plotat 88], [Smolka 89].

${ }^{4}$ Since we allow cyclicity, unrestricted deductive closure algoritlıns (cf. e.g. [Kasper/Rounds 86] and [Kasper/Rounds 90]) cannot be applied. 
value languages.

Moreover, this closure construction is the direct prooftheoretic correlate of the congruence closure algorithm (cf. (Nelson/Oppen 80]), if it were used for testing satisfiability of finite sets of literals in $H_{A V}^{\circ}$. As it is shown there, the congruence closure algorithm can be used to test consistency if the terms of the equations are represented as labeled graphs and the equations as a relation on the nodes of that graph.

On the basis of the algorith $m$ for deciding satisfiability of finite sets of formulas we then show the completencss and decidability of $H_{A V}^{\circ}$.

\section{Attribute-Value Languages}

In this section we define the type of language we want to consider and introduce some additional notation.

\subsection{Syntax}

2.1. DEFINITION. A quantifier-free attribute-value language $\left(\right.$ Le $\mathcal{L}_{A V}^{0}$ ) consists of the logical connectives $\perp$ (false), $\sim$ (negation), $\supset$ (implication), the equality symbol $\approx$ and the parentheses (,). The nonlogical vocabulary is given by a finite set of constants $\mathcal{C}$ and a finite set of unary partial function symbols $F_{1}\left(C \cap F_{1}=\theta\right)$.

2.2. DEFINITION. The class of terms $(\mathcal{T})$ of $L$ is recursively defined as follows: each constant is a term; if $f$ is a funclion symbol and $r$ is a term, then $f r$ is a term.

2.3. DEFINITION. The set of atomic formulas' of $L$ is $\left\{r_{1} \approx \tau_{2} \mid \tau_{1}, \tau_{2} \epsilon T\right\} \cup\{\perp\}$.

2.4. DEFINITION. The formulas of $L$ are the atomic formulas and, whenever $\phi$ and $\psi$ are formulas, then so are $(\sim \phi)$ and $(\phi \supset \psi)$.

2.6. DEFINITION. If $\alpha$ is a well-formed expression (lerm or formula), then $\alpha\left[r_{1} / r_{2}\right]$ is used to designatc an expression obtained from $\alpha$ by replacing some (possibly all or none) occurrences of $\tau_{1}$ in $\alpha$ by $\tau_{2}$.

We assume that the connectives $\vee$ (disjunction), \& (conjunction) and $\equiv$ (equivalence) are introduced by their usual definitions, Furthermore, we write sometimes $\tau_{1} \not \approx \tau_{2}$ instead of $\sim r_{1} \approx r_{2}$ and drop the parentheses according to the usual conventions. ${ }^{6}$

\subsection{Semantics}

$A$ model for $L$ consists of a nonemply universe $U$ and an interprepation function $\Im$. Since not every term denotes an element in $\boldsymbol{\mu}$ if the function symbols are interpreted as unary partial functions, we generalize the partiality of the denotation by asouming that $\mathbf{a}$ itself is a partial function. Thus in general not

\footnotetext{
Cf. also [Statiman 77].

We drop the outermost brackets, assume that the conncctives have the precedence $\sim>\& \vee>\supset, \equiv$ and are left associative.
}

all of the constants and function symbols are interpreted by $\boldsymbol{9}$. Redundancies which result from the fact that non-interpreted function symbols and function symbols interpreted is emply functions are then regarded as distinct are removed by requiring these partial functions to be nonempty. Suppose $[X \rightarrow Y](p)$ designates the set of all (partial) functions from $X$ to $Y$, then a model is defined as follows:

2.6. DEFINITION. A model for $L$ is a pair $M=\langle U, \Im\rangle$, consisting of a nonempty set $U$ and an interpretation function $\Im=\Im_{C} \cup \Im_{F_{1}}$, such that

$$
\begin{aligned}
& \text { (i) } \Im_{c \epsilon}[\mathcal{C} \mapsto U]_{p} \\
& \text { (ii) } \Im_{F_{1}}\left[F_{1} \mapsto[U \mapsto U]_{p}\right]_{p} \\
& \text { (iii) } \forall f_{c} F_{1}(f \subset \operatorname{Dom}(\Im) \rightarrow \Im(f) \neq \emptyset) .
\end{aligned}
$$

The (partial) denotation function for terms $\bar{\Im}\left(\overline{\mathcal{S}} \in\left[\tau \mapsto u_{p}\right)\right.$ induced by $\Im$ is defined as follows:?

2.7. DEWINITION. For every $c c \mathcal{C}$ and $f r c \mathcal{T}\left(f \in F_{1}\right)$,

$$
\begin{aligned}
& \bar{\Im}(c)= \begin{cases}\Im(c) & \text { if } c \epsilon \operatorname{Dom}(\Im) \\
\text { undefined } & \text { otherwise }\end{cases} \\
& \bar{\Im}(f r)= \begin{cases}\Im(f)(\bar{\Im}(\tau)) & \text { if } f \epsilon \operatorname{Dom}(\Im) \wedge \bar{\Im}(r) \text { defined } \wedge \\
\text { undefined } & \begin{array}{l}
\Im(r) \epsilon \operatorname{Dom}(\Im(f)) \\
\text { otherwise. }
\end{array}\end{cases}
\end{aligned}
$$

2.8. DEFINITION. The satisfaction relation between models $M$ and formulas $\phi\left(F_{M} \phi\right.$, read: $M$ satisfies $\phi, M$ is a model of $\phi, \phi$ is true in $M)$ is defined recursively:

$$
\begin{aligned}
& \vDash_{M} \perp \\
& \vDash_{M} \tau \approx r^{\prime} \leftrightarrow \Im(\tau), \Im\left(r^{\prime}\right) \text { defined } \wedge \Im(\tau)=\Im\left(r^{\prime}\right) \\
& F_{M} \sim \phi \rightarrow \neg\left(F_{M} \phi\right) \\
& \vDash_{M} \psi \supset x \rightarrow \vDash_{M} \psi \rightarrow \vDash_{M} x .
\end{aligned}
$$

A formula $\phi$ is valid $(=\phi)$, iff $\phi$ is true in all models. A formula $\phi$ is satisfiable, iff it has at least one model. Given a set of formulas $\Gamma$, we say that $M$ satisfies $\Gamma\left(F_{M} \Gamma\right)$, if $M$ satisfies eacli formula $\phi$ in $\Gamma$. $\Gamma$ is satisfiable, if there is a model that satisfies each formula in $\Gamma$. $\phi$ is logical consequence of $\Gamma$ $(\Gamma \vDash \phi)$, iff every model that satisfies $\Gamma$ is a model of $\phi$.

\section{The System $H_{A V}^{0}$}

In this section we describe an axiomalic or Hilbert type system $I_{A V}^{0}$ for quantifier-free attribute-value languages $L$. We give a decision procedure for the satisfiability of finite sets of formulas and show the completeness and decidability of $H_{A V}^{0}$ on the basis of that procedure.

\subsection{Axioms and Inference Rules}

If $L$ is a fixed attribute-value language, then the system consiats of a traditional axiomatic propositional calculus for $L$ and two additional equality axioms. For any formulas $\phi, \psi, x$, terms

\footnotetext{
${ }^{7}$ In the text following the definition we drop the overline.
} 
$r, r^{\prime}$, and every sequence of functors $\sigma\left(\sigma c F_{i}^{*}\right)$ of $L$ the formulas under $A 1$ - A4 are propositional axioms and the formulas under $E 1$ and E2 are equality axioms. ${ }^{9}$ 'The Modus l'onens (MP) is the only inference rule. ${ }^{10}$

$$
\begin{array}{ll}
\text { A1 } & \vdash \sim \perp \\
\text { A2 } & \vdash \phi \supset(\psi \supset \phi) \\
\text { A3 } & \vdash(\phi \supset(\psi \supset \chi)) \supset((\phi \supset \psi) \supset(\phi \supset \chi)) \\
\text { A4 } \vdash(\sim \phi \supset \sim \psi) \supset(\psi \supset \phi) \\
\text { E1 } \vdash \sigma r \approx r^{\prime} \supset r \approx r \\
\text { E2 } \vdash r \approx r^{\prime} \supset\left(\phi \supset \phi\left[r / r^{\prime}\right]\right) \\
\text { MP } \phi \supset \psi \wedge \phi \vdash \psi
\end{array}
$$

A formula $\phi$ is derivable from a set of formulas $\Gamma(\Gamma \vdash \phi)$, if there is a finite sequence of formulas $\phi_{1} \ldots \phi_{n}$ such that $\phi_{n}=\phi$ and every $\phi_{i}$ is an axiom, one of the formulas in $\Gamma$ or follows by MP from two previous formulas of the sequence. $\phi$ is a theorem $(\vdash \phi)$, iff $\phi$ is derivable from the empty set. $\Delta$ is derivable from $\Gamma(\Gamma \vdash \Delta)$, iff each formula of $\Delta$ is derivable from $\Gamma$. $\Gamma$ and $\Delta$ are deduclively equivalent $(\Gamma \dashv \Delta)$, if $\Gamma \vdash \Delta$ and $\Delta \vdash \Gamma$.

The system is sound: ${ }^{11}$

\subsection{THEOREM. For every formula $\phi:$ If $\downarrow$, then $=\phi$.}

Beside this weak version also the strang soundness theorem is provable for $H_{A V}^{0}$ :

3.2. THEOREM. For every set of formulas $\Gamma$ and every formula $\phi$ : If $\Gamma \vdash \phi$, then $\Gamma \vDash \phi$.

\subsection{Satisfiability}

We now prove

3.3. THEOREM. The salisfiabilily of a finile set of formulas $\Gamma$ is decidable.

by providing a terminating procedure: Pirst the conjunction of all formulas in $\Gamma$ (denoted by $\wedge \Gamma$ ) is converted into disjunctive normal form $(D N F)$ using the well-known standard techniques. Then $\Lambda \Gamma$ is equivalent with a $D N F$

$\vdash \wedge \Gamma \equiv\left(\phi_{1}^{1} \& \phi_{2}^{1} \& \ldots \& \phi_{k_{1}}^{1}\right) \vee\left(\phi_{1}^{2} \& \ldots \& \phi_{k_{2}}^{2}\right) \vee \ldots \vee\left(\phi_{1}^{n} \& \ldots \& \phi_{k_{n}}^{n}\right)$

where the conjuncts $\phi_{j}^{i}\left(i=1, \ldots, n_{i} j=1, \ldots, k_{i}\right)$ are either atomic formulas or negations of atomic formulas, henceforth called literals. By the definition of the satisfiability we get:

\footnotetext{
${ }^{8}$ Cf. e.g. [Church 56].

${ }^{9}$ Axiom El restricts the reflexivity of identity to denoting terns: if a term denotes, then also its sublerms do (cf. the definition of $\boldsymbol{\Im}$ ). Thus equality is not a reflexive, but only a subtern reflexive relation. ${ }^{10}$ If (i.) constant-consistency and (ii.) constant/complexconsistency are to be guaranteed for a set of atomic values $V(V \subseteq \mathcal{C}$ ), for each $a, b c V(a \neq b)$ and $f c F_{1}$, axioms of the form (i.) $\vdash a \neq b$ and (ii.) $\vdash f a \neq f a$ have to be added (a finite set). If also acyclicity has to be ensured, sxioms of the form (iii.) $\vdash \sigma \tau \approx \tau$, with $\sigma \in F_{1}^{+}, \tau \in \mathcal{T}$, have to be added. Although this set is infinite, we only need a finite subset for the satisfiability test and for docidability (see below).

"For the propositional calculus of. the standard proofs. For axioms E1 and E2 cf. [Johnson 88].
}

3.4. LEMMA. Let $\bigwedge S^{1} \vee \wedge S^{2} \vee \ldots \vee \wedge S^{n}$ be a $D N F$ of $\Lambda \Gamma$ consisting of conjunctions $\Lambda S^{i}$ of the literals in $S^{i}$, then $\Lambda \Gamma$ is satisfiable, iff at lcast one disjunct $\bigwedge S^{i}$ is satisfiable.

We complete the proof of Theorem 3.3 by an algorithm that converts a finite set of literals $S^{i}$ into a deductively equivalent set of literals in normal form $S_{\nu}^{i}$ which is satisfiable iff it is not equal to $\{1\}$.

\subsubsection{A Normal Form for Sets of Literals}

The normal form is constructed by closing $S$ deductively by those equations whose terms are subterms of the terms occurring in $S$. For the construction we use the following derived rules:

$$
\begin{array}{lll}
\text { R1 } & \sigma r \approx r^{\prime} \vdash \tau \approx \tau & \text { Subterm Reflexivity } \\
\text { R2 } & \tau \approx r^{\prime} \wedge \phi \vdash \phi\left[r / \tau^{\prime}\right] & \text { Substilutivity } \\
\text { R3 } & r \approx \tau^{\prime} \vdash \tau^{\prime} \approx \tau & \text { Symmetry. }
\end{array}
$$

We get $R_{1}$ and $R 2$ from E1 and E2 by the deduction theorem. $R .3$ is derivable from $R 1$ and $R 2$, since we get from $\tau \approx \tau^{\prime}$ first $\tau \approx \tau$ by $\mathrm{R} 1$ and then $\tau^{\prime} \approx \tau$ by $R 2$.

If $T_{S}$ denotes the set of terms occurring in the formulas of $S$ $\left(T_{S}=\left\{\tau, \tau^{\prime} \mid(\sim) \tau \approx \tau^{\prime} \epsilon S\right\}\right)$, and $\operatorname{SUB}\left(T_{S}\right)$ denotes the set of all sublerms of the terms in $\mathcal{T}_{S}{ }^{12}$

$$
\operatorname{SUB}\left(\mathcal{T}_{S}\right)=\left\{\tau \mid \sigma \tau \epsilon \mathcal{T}_{S}, \text { with } \sigma \epsilon F_{1}^{*}\right\}
$$

then the normal form is constructed according to the following inductive definition.

3.5. DEFINITION. For a given set of literals $S$ we define a seqnence of sets $S_{i}(i \geq 0)$ by induction:

With $S_{0}^{\prime}=S \cup\left\{r^{\prime} \approx \tau \mid \tau \approx \tau^{\prime} \epsilon S\right\}$,

$S_{0}= \begin{cases}\{\perp\} & \text { if } \perp \epsilon S ; \text { otherwise } \\ S_{0}^{\prime} \cup\left\{\tau \approx \tau \mid \sigma \tau \approx r^{\prime} \epsilon S_{0}^{\prime}\right\}\end{cases}$

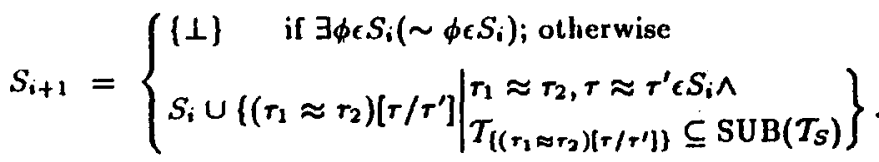

Since $S_{i} \subseteq S_{i+1}$, for $S_{i+1} \neq\{1\}$, the construction terminates on the basis of the subterm condition either with a finite set of literals or with $\{1\}$. If each term of the equations in $S_{i+1}$ is a subterm of the terms in $\mathcal{T}_{S}$, no term of the equations in $S_{i+1}$ can be longer than the longest term in $\tau_{S}$.

EXAMPLE 1. Assume that $L$ consists of the constants $a, b, c, e$ and the function symbols $f, g, h, m, n, p$. Then, for the set of literals

$$
S=\left\{\begin{array}{l}
g e \approx p m b, e \approx m e, m b \approx n g f f c, c \approx a, \\
g a \approx h a, a \approx f f a, n g f f a \neq e
\end{array}\right\}
$$

the following sequence of sets is constructed. We represent the equations of a set $S_{i}$ by the system of sets of equivalent terms induced by $S_{i}$. I.e.: If $\Theta$ is a set of terms under $S_{i}$ and

\footnotetext{
${ }^{12} \mathcal{T}_{S} \subseteq \operatorname{SUB}\left(\mathcal{T}_{S}\right)$ loolds by definition.
} 
$r, r^{\prime} \epsilon \Theta$, then $r \approx r^{\prime} c S_{i}$. Furthermore, we mark by an arrow that a set under $S_{i}$ is also induced (without modifications) by the equations in $S_{i+1}$.

\begin{tabular}{|c|c|c|}
\hline$S_{0}$ & $S_{1}$ & $S_{2}=S_{\nu}$ \\
\hline$n g f f a \not e$ & $\rightarrow$ & $\rightarrow$ \\
\hline$\{e, m e\}$ & $\rightarrow$ & $\rightarrow$ \\
\hline $\begin{array}{r}\{b\} \\
\{c, a\}\end{array}$ & $\rightarrow$ & $\rightarrow$ \\
\hline $\left.\begin{array}{c}\{a, f f a\} \\
\{f f c\}\end{array}\right\}$ & $\{c, a, f f a, f f c\}$ & $\rightarrow$ \\
\hline$\{g e, p m b\}$ & $\rightarrow$ & $\rightarrow$ \\
\hline$\{m b, n g f f c\}$ & $\{m b, n g f f c, n g f f a\}$ & $\rightarrow$ \\
\hline $\left.\begin{array}{l}\{f c\} \\
\{f a\}\end{array}\right\}$ & $\{f c, f a\}$ & $\rightarrow$ \\
\hline $\begin{array}{c}\{g f f c\} \\
\{g a, h a\}\end{array}$ & $\left.\begin{array}{c}\{g f f c, g f f a\} \\
\{g a, h a, g f f a\}\end{array}\right\}$ & $\{g f f c, g f f a, g a, h a\}$ \\
\hline
\end{tabular}

3.6. DEFINITION. Let $S_{\nu}=S_{t}$; with $t=\min \left\{i \mid S_{i}=S_{i+1}\right\}$.

3.7. LEMMA. For $S_{\nu}$ holds: $S$ t $S_{\nu}$.

PROOF. If $S_{\nu} \neq\{\perp\}$, then $S$ and $S_{\nu}$ are deductively equivalent, since $S$ is a subset of $S_{\nu}$ and $S_{\nu}$ only contains formulas derivable from $S$. For $S_{\nu}=\{1\}$ the same holds for $S_{\nu-1}$. Since $S_{\nu-1}$ is inconsistent, $S$ is deductively equivalent with $(\perp)$. 口

Note that for each equation in $S_{i}\left(S_{i} \neq(\perp\}\right)$ there is a proof from $S$ with the subterm properly, as defined below. This follows from the subterm condition in the inductive construction.

3.8. DEFINITION. A proof of an equation from $S$ has the subterm property, iff each term occurring in the equations of that proof is a subterm of the terms in $\tau_{S}$, i.e. an element of $\operatorname{SUB}\left(\mathcal{T}_{S}\right)$.

So, if $S$ is not trivially inconsistent $(\perp$ not in $S$ ), the construction terminates with $\{\perp\}$, since there exists a proof of an equation from $S$ with the subterm properly, whose negation is in $S$.

EXAMPLE 2. For the inconsistent set $S^{\prime}=S \cup\{g m m e \not z n h f f a\}$ the construction terminates after 4 steps $\left(S_{1}^{\prime}=\{1\}\right)$, since there is a proof of $g m m e \approx p n h f f a$ from $S^{\prime}$ with the subterm property of depth 3 .

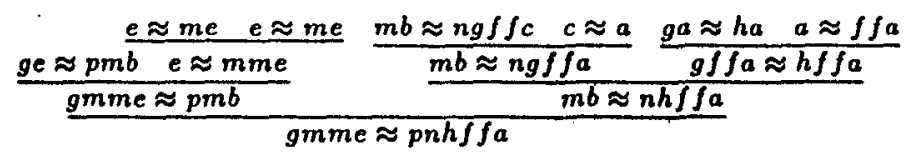

The deductive closure construction restricted by the subterm property is a proof-theoretic simulation of the congruence closure algorithm (cf. [Nelson/Oppen 80$]^{13}$ ), if used for testing satisfiability of finite sets of literals in $I_{A V}^{0}$. Strictly speaking, if

i. the congruence closure algorithm is weakened for partial functions,

ii. $S$ is not trivially inconsistent ( $\perp$ not in $S$ ), and

iii. the failure in the induction step of 3.5. is overrnled,

\footnotetext{
${ }^{13}$ Cf. also [Gallier 87].
}

then $\tau \approx r^{\prime}$ is in $S_{\nu}$ iff the nodes which represent the terms $\tau$ and $\tau^{\prime}$ in the graph constructed for $S$ are congruent. ${ }^{14}$ Moreover, for unary partial functions the algorithm is simpler, since the arity does not have to be controlled.

3.9. LEMMA. The set of all equations in $S_{\nu}$ is closed under subterm reflexivity, symmetry and transitivily.

PROOF. For $S_{\nu}=\{1\}$ trivial. If $S_{\nu} \neq\{1\}$, then $S_{\nu}$ is closed under subterm reflexivity and symmetry, since these properties are inherited from $S_{0}$ to its successor sets. $S_{\nu}$ is closed under transitivity, since we first get $\tau_{3} C \operatorname{SUB}\left(\mathcal{T}_{S}\right)$ from $r_{1} \approx r_{2}, r_{2} \approx r_{3} \epsilon S_{\nu}$ and then according to the construction also $r_{1} \approx \tau_{2}\left[\tau_{2} / \tau_{3}\right] c S_{\nu+1}=S_{\nu}$, with $\tau_{2}\left[\tau_{2} / \tau_{3}\right]=\tau_{3}$.

\subsubsection{Satisfiability of Sets of Literals}

For the proof that the satisfiability of a finite set of literals is decidable we first show that a set of literals in normal form is satisfiable, iff the set is not equal to $\{\perp\}$. For $S_{\nu}=\{\perp\}$ we get trivially:

$$
\text { 3.10. LEMMA. } S_{\nu}=\{\perp\} \rightarrow \neg \exists M\left(\vDash F_{M} S_{\nu}\right) \text {. }
$$

Otherwise we can show the satisfiability of $S_{\nu}$ by the construction of a canonical model that satisfies $S_{\nu}$.

Let $E_{\nu}$ be the set of all (nonnegated) equations in $S_{\nu}, \mathcal{T}_{E_{\nu}}$ the set of terms occurring in $E_{\nu}$ and $\approx_{E_{\nu}}$ the relation induced by $E_{\nu}$ on $\tau_{E_{\nu}}\left(\left\{\left\langle\tau, \tau^{\prime}\right\rangle \mid \tau \approx r^{\prime} \epsilon E_{\nu}\right\}\right)$. Then, we choose as the universe of the canonical model $M_{\nu}=\left\langle U_{\nu}, \Im_{\nu}\right\rangle$ the set of all equivalence classes of $\approx_{E_{\nu}}$ on $T_{E_{\nu}}$, if $\mathcal{T}_{E_{\nu}} \neq \emptyset$. By Lemma 3.9 this set exists. If $S_{\nu}$ contains no (unnegated) equation, we set $u_{\nu}=\{\emptyset]$, since the universe has to be nonempty.

3.11. DEFINITION. For a set of literals $S_{\nu}$ in normal form, the canonical term model for $S_{\nu}$ is given by the pair $M_{\nu}=\left\langle u_{\nu}, \Im_{\nu}\right\rangle$, consisting of the universe

$$
u_{\nu}= \begin{cases}\mathcal{T}_{E_{\nu}} / \approx_{E_{\nu}} & \text { if } \mathcal{T}_{E_{\nu} \neq \emptyset} \neq \emptyset \\ \{\theta\} & \text { otherwise }\end{cases}
$$

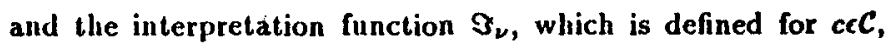
$f \epsilon F_{1}$ and $[r] \epsilon U_{\nu}$ by: $:^{15}$

$$
\begin{aligned}
& \Im_{\mathcal{C}}(c)= \begin{cases}{[c]} & \text { if } c \epsilon \mathcal{T}_{E_{\nu}} \\
\text { undefined } & \text { otherwise }\end{cases} \\
& \Im_{F_{1}}(f)([\tau])= \begin{cases}{\left[f \tau^{\prime}\right]} & \text { if } \tau^{\prime} c[\tau] \text { and } f \tau^{\prime} c \mathcal{T}_{E_{\nu}} \\
\text { undefined } & \text { otherwise. }\end{cases}
\end{aligned}
$$

It follows from the definition that $\Im_{\nu}$ is a partial function. Suppose further for $\Im_{F_{1}}(f)$ that $\left[r_{1}\right]=\left[r_{2}\right]$ and that $\Im_{F_{1}}(f)\left(\left[r_{1}\right]\right)$ is defined. Then

$$
\Im_{F_{1}}(f)\left(\left[r_{1}\right]\right)=\Im_{F_{1}}(f)\left(\left[r_{2}\right]\right) .
$$

For this, suppose $\Im_{F_{1}}(f)\left(\left[r_{1}\right]\right)=\left[f \tau^{\prime}\right]$, with $\tau^{\prime} c\left[r_{1}\right]$. Since $\approx E_{v}$ is an equivalence relation we get $r^{\prime} \in\left[r_{2}\right]$ and thus $\Im_{F_{1}}(f)\left(\left[\tau_{2}\right]\right)=\left[f \tau^{\prime}\right]$

\footnotetext{
${ }^{14} \mathrm{Cr}$. [Wedekind 90].

${ }^{15}$ We drop the $\approx_{E_{\mu}}$-index of the equivalence classes.
} 
EXAMPLE 3. The canonical model for $S$ of Example 1 which is constructed using $S_{2}=S_{\nu}$ is given by:

$$
\begin{aligned}
& U_{\nu}=\left\{\begin{array}{c}
\{c, m c\},\{b\},\left\{c, a, \int f a, f \int c\right\}, \\
\{g e, p m b\},\{m b, n g f f c, n g f f a\}, \\
\{f c, f a\},\left\{g f \int c, g f \int a, g a, h a\right\}
\end{array}\right\} \\
& \Im_{\nu}(e)=[c] \\
& \Im_{\nu}(b)=[b] \\
& \left.\begin{array}{l}
\Im_{\nu}(c) \\
\Im_{\nu}(a)
\end{array}\right\}=[c] \\
& \Im_{\nu}(f)=\left\{\begin{array}{c}
([a],[f a]\rangle, \\
\langle[f a],[f f a]\rangle
\end{array}\right\} \\
& \Im_{\nu}(m)=\left\{\begin{array}{c}
([e],[m e]\rangle, \\
([b],[m b]\rangle
\end{array}\right\} \\
& \Im_{\nu}(g)=\left\{\begin{array}{c}
\langle[e],[g e]\rangle, \\
\langle[a],[g a]\rangle
\end{array}\right\} \\
& \Im_{\nu}(h)=\{\langle[a],[h a]\rangle\} \\
& \Im_{\nu}(n)=\{\langle[g a],[n g f f c]\rangle\} \\
& \Im_{\nu}(p)=\{\langle[m b],[p m b]\rangle\} .
\end{aligned}
$$

For each term $\tau$ in $\mathcal{T}_{E_{\nu}}$ it follows from the definition of $\Im_{\mathcal{C}}$ and $\Im_{F_{2}}: \Im_{\nu}(r)=[r]$.

By the following lemma we show in addition that the domain of $\Im_{\nu}$ restricted to $T_{S_{\nu}}$ is equal to $T_{E_{\nu}}$.

3.12. LEMM $\Lambda$. For each term $\tau$ in $\mathcal{T}_{S_{\nu}}$ : If $\Im_{\nu}$ is defined for $\tau$, then $\Im_{\nu}(\tau)=[r]$, with $\tau \in \mathcal{T}_{E_{\nu}}$.

PROOF. (By induction on the length of $\tau$.) Suppose first that $\boldsymbol{S}_{\nu}$ is defined for $r$. For every constant $c$ it follows from the definition of $\Im_{\mathcal{C}}$ that $\Im_{c}(c)=[c]$, with $c \in \mathcal{T}_{E_{\nu}}$. Assume for $f \tau$ by inductive hypothesis $\Im_{\nu}(\tau)=[\tau]$, with $\tau \in T_{E_{\nu}}$, then it follows from the definition of $\Im_{F_{1}}(f)$ that $\Im_{F_{1}}(f)([\tau])=\left[\int \tau^{\prime}\right]$, with $f r^{\prime} c T_{E_{\nu}}$ and $\tau^{\prime} \epsilon[r]$. Since $\tau^{\prime}$ is a subterm of $f r^{\prime}$, we first get $r^{\prime} \epsilon T_{E_{\nu}}$ and by Lemma $3.9 f r^{\prime} \approx f r^{\prime}, r^{\prime} \approx r \epsilon S_{\nu}$. Because of freSUB $\left(T_{S}\right)$, then also $f \tau \approx f \tau \epsilon S_{\nu}$. So, $f \tau$ must also be in $T_{E_{\nu}}$ and hence $\Im_{F_{1}}(f)([\tau])=[\delta \tau]$.

Next we show for the model $M_{\nu}$ :

3.13. LEMMA. $S_{\nu} \neq\{1\} \rightarrow \neq F_{M_{\nu}} S_{\nu}$.

PROOF. (We prove $\models M_{\nu} \phi$, for every $\phi$ in $S_{\nu}$ by induclion on the structure of $\phi$.)

$\perp$ is not element of $S_{\nu}$. If $\perp$ were in $S_{\nu}$, we would get by the definition of $S_{\nu} S_{\nu}=\{\perp\}$ which contradicts our assumption.

For $\phi=\sim \perp,=_{M_{\nu}} \sim \perp$ holds trivially.

Suppose $\phi=\tau \approx \tau^{\prime}$, then $\tau, \tau^{\prime}$ are in $\mathcal{T}_{E_{\nu}}, \mathcal{S}_{\nu}$ is defined for $r$ and $r^{\prime}$, and $\Im_{\nu}(\tau)=[r], \Im_{\nu}\left(r^{\prime}\right)=\left[r^{\prime}\right]$. Because of $\tau \approx$ $r^{\prime} \epsilon S_{\nu}$, it follows that $[r]=\left[r^{\prime}\right]$. So $\sigma_{\nu}(r)=\sigma_{\nu}\left(r^{\prime}\right)$ and hence $F_{M_{\nu}} \tau \approx \tau^{\prime}$.

Assume that $\phi$ is $\sim\left(\tau \approx r^{\prime}\right)$. If $\tau \approx \tau^{\prime}$ were satisfied by $M_{\nu}$, $\Im_{\nu}(\tau)$ would be equal to $\Im_{\nu}\left(\tau^{\prime}\right)$. By Lemma 3.12 we would then get $\Im_{\nu}(\tau)=[r]$ and $\Im_{\nu}\left(r^{\prime}\right)=\left[r^{\prime}\right]$, with $\tau, r^{\prime} \in \mathcal{T}_{E_{\nu}}$. Since $\approx_{E_{\nu}}$ is an equivalence relation on $T_{E_{\nu}}, \tau \approx r^{\prime} c S_{\nu}$ would follow from $[r]=\left[r^{\prime}\right]$, and, contradicting the assumption, we would get $S_{\nu}=\{\perp\}$ by the definition of $S_{\nu}$.

It can be easily shown that $M_{\nu}$ is a unique (up to isomorphism) minimal model for $S_{\nu}{ }^{16}$ Strictly speaking, if $M$ is a model for

\footnotetext{
${ }^{16}$ It can be verificd very easily by using this fact that we need to add to a set of literals $S$ only a finite number of axions to ensure the acyclicity. All axioms of the form or $\neq \tau\left(\sigma e F_{1}^{+}, \tau e T\right)$, with $|\sigma r| \leq$ $\left|\operatorname{SUB}\left(T_{E}\right)\right|$, are e.g. more than enough, since from a consistent but cyclic set of literals $S$ must follow an equation $\sigma \tau \approx \tau\left(\sigma e F_{1}^{+}, \tau \in \mathcal{T}\right)$, with $|\sigma r| \leq\left|U_{\nu}\right|$, and $\left|\mathcal{U}_{\nu}\right| \leq\left|\operatorname{SUB}\left(\mathcal{T}_{E}\right)\right|$ holds by the construction of $\boldsymbol{u}$.
}

$S_{\nu}$ homomorphic to $M_{\nu}$, then every minimal submodel of $M$ that satisfies $S_{\nu}$ is isomorphic to $M_{\nu}$.

From the two lemmata above it follows first that the satisfiability of sets of formulas in normal form is decidable:

$$
S_{\nu} \neq\{\perp\} \leftrightarrow \exists M\left(\models_{M} S_{\nu}\right)
$$

Since $S_{\nu}$ and $S$ are deductively equivalent, we can establish by the following lemma that the satisfiability of arbitrary finite sets of literals $S$ is decidable.

3.14. LEMMA. $S_{\nu} \neq\{\perp\} \leftrightarrow \exists M\left(\models_{M} S\right)$.

PROOF. $(\rightarrow)$ If $S_{\nu} \neq\{\perp\}$, we know by Lemma 3.13 that $M_{\nu}$ is a model for $S_{\nu}$. Then, by the soundness $S_{\nu}+S \rightarrow \forall M(\vDash M$ $S_{\nu} \rightarrow=_{M} S$ ). Since $S$ is derivable from $S_{\nu}$, it follows $\models_{M_{\nu}} S$ and thus $S_{\nu} \neq\{1\} \rightarrow \exists M\left(\left.\right|_{M} S\right)$.

$(-)$ If $S_{\nu}=\{1\}$, then for each model $M \nvdash_{M} S_{\nu}$. From the soundness we get $S \vdash S_{\nu} \rightarrow \forall M\left(\vDash_{M} S \rightarrow \vDash_{M} S_{\nu}\right)$. Since $S_{\nu}$ is derivable from $S$, it follows $\forall M\left(\not \neq_{M} S_{\nu} \rightarrow \not \nvdash_{M} S\right)$ and hence $S_{\nu}=\{1\} \rightarrow \forall M\left(\not \neq_{M} S\right)$.

\subsection{Completeness and Decidability}

Using the procedure for dcciding satisfiability we can easily show the completeness and decidability of $H_{A V}^{0}$.

3.15. THEOREM. For every finite set of formulas $\Gamma$, and for cach formula $\phi:$ If $\Gamma \vDash \phi$, then $\Gamma \vdash \phi$.

PROOF. By definition $\phi$ is a logical consequence of $\Gamma$, if $\Gamma \cup\{\sim \phi\}$ is unsatisfiable. Using the equivalences of Theorem 3.3 , we first get:

$$
\Gamma \cup\{\sim \phi\} \Vdash\{\Lambda(\Gamma \cup\{\sim \phi\})\}
$$

Suppose, that $\wedge S^{1} \vee \ldots \vee \wedge S^{n}$ is a $D N F$ of $\wedge(\Gamma \cup\{\sim \phi\})$, then

$$
\Gamma \cup\{\sim \phi\} \Vdash\left\{\Lambda^{1} \vee \ldots \vee \wedge S^{n}\right\}
$$

and by the decision procedure

$$
\not \models \Gamma \cup\{\sim \phi\} \leftrightarrow S_{\nu}^{l}=\{\perp\} \wedge \ldots \wedge S_{\nu}^{n}=\{\perp\} .
$$

If $\Gamma \cup\{\sim \phi\}$ is unsatisfiable, it follows that $\Gamma \cup\{\sim \phi\}$ th $\{\perp\}$, since each $S^{i}$ is deductively equivalent with $\{1\}$. From $\Gamma \cup\{\sim \phi\} \vdash \perp$ it follows by the deduction theorem firat $\Gamma \vdash \sim \phi \supset \perp$ and thus $\Gamma \vdash \sim \perp \supset \phi$. From $\Gamma \vdash \sim \perp \supset \phi$ and $\Gamma \vdash \sim \perp$ by MP then $\Gamma \vdash \phi$.

3.16. COROLLARY. For every finile set of formulas $r$ and each formula $\phi, \Gamma \vdash \phi$ is decidable.

PROOF. By the completeness and soundness we know $\Gamma \vdash \phi \rightarrow$ $\Gamma \models \phi$. Since $\phi$ is a logical consequence of $\Gamma$, if $\nvdash \Gamma \cup\{\sim \phi\}$, we can decide $\Gamma \vdash \phi$ by the procedure for deciding $\forall \Gamma \cup\{\sim \phi\}$.

\section{Acknowledgments}

The author has been supported during the writing of the submitted draft version of this paper by the EEC Esprit project 
DYANA at the Institut für maschinefle Sprachverarbeitung, Universität Stuttgart. The autlıor would like to thank Jochen Dörre, Mark Johnson, Ilans Kanp, Ron Kaplan, Paul King, John Maxwell and Stefan Momma as well as an anonymous reviewer for their comments on earlicr versions of this paper. All remaining errors are of course my own.

\section{References}

[Beierle/Pletat 88] Beierle, C., U. Pletat: Feature Graphs and Abstract Data Types: A Unifying Approach. Proceedings of COLING 88, Budapest 1988

[Church 56] Church, A.: Introduction to Mathemalical Logic, Princeton 1956

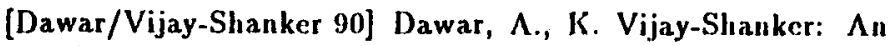
Interpretation of Negation in Fealure Siructure Descriptions. Computational Linguistics, 16, 11-21

[Gallier 87] Galljer, J.: Logic for Computer Science: Foundations of Automatic Theorem P'roving. New York 1987

[Johnson 88] Johnson, M.: Attribute-Value Logic and the Theory of Grammar. PhD thesis, Stanford University, Stanford 1988

[Kasper/Rounds 86] Kasper, R., W. Rounds: 1 logical Somantics for Feature Structures. Proccedings of the 2 th Annual Meeting of the $A C L$, New York 1986
[Kasper/Rounds 90] Kasper, R., W. Rounds: The Logic of Unification in Grammar. Linguistics and Philosophy, 13, $35-58$

[Knuth/Bendix 70] Knuth, D., P. Bendix: Simple Word Problems in Universal Algebra. In: J. Leech (ed.): Compulational Problems in Universal Algebra, Elmsford, N.Y., 1970

[Kreisel/Tait 61] Kreisel, G., W. Tait: Finite Definability of Number-theoretic Functions and Parametric Completeness of Equational Calculi. Z. Math. Logik Grundlagen Math., 7, 28-38.

[Langholm 89] Langholm, T.: How to Say No with Feature Structures. COSMOS-Report No. 13, Oslo 1989

[Nelson/Oppen 80] Nelson, G., D. Oppen: Fast Decision Procedures Based on Congruence Closure. ACM, 27, 356-364

[Sinolka 89] Smolka, G.: Feature Constraint Logics for Unification Grammars. IWBS Report 93, Stuttgart 1989

[Statman 74] Statman, R.: Structural Complexity of Proofs. PhD thesis, Stanford University, Stanford 1974

[Statman 77] Statman, R.: Herbrand's Theorem and Gentzen's Notion of a Direct Proof. In: J. Barwise (ed.): Handbook of Malhematical Logic, Amsterdam 1977

[Wedekind 90] Wedekind, J.: Unifikationsgrammatiken und ihre Logik. PhD thesis, Universität Stuttgart, Stuttgart 1990 